\title{
NOMENKLATORISCHE BERICHTIGUNG VON OSTRYO-QUERCETUM PUBESCENTIS (HORVAT 1959) TRINAJSTIĆ 1977
}

\author{
Livio POLDINI*
}

\begin{abstract}
In accordance with the codex of phytocoenological nomenclature, we have corrected the name of following plant community: Ostryo-Quercetum pubescentis (Horvat 1959) Trinajstič 1977 with the new name Aristolochio luteae-Quercetum pubescentis (Horvat 1959) Poldini nom. nov. hoc. loco.

Key words: International Code of Phytosociological Nomenclature, syntaxa

\section{Izvleček}

V skladu s kodeksom fitocenološke nomenklature smo popravili ime rastlinske združbe: Ostryo-Quercetum pubescentis (Horvat 1959) Trinajstič 1977 z novim imenom Aristolochio luteae-Quercetum pubescentis (Horvat 1959) Poldini nom. nov. hoc. loco.
\end{abstract}

Ključne besede: Mednarodni kodeks fitosociološke nomenklature, sintakson

Der verbreiteste epimediterrane Hopfenbuchen-Flaumeichenwald im küstennahen Teil der balkanischen Halbinsel wurde als „Ostryeto-Seslerietum autumnalis quercetosum pubescentis Horvat et Horvatić 1946 prov." bezeichnet. In der Arbeit werden nur die häufigsten Arten aber keine Aufnahmen angeführt; eben da wird auch die Gliederung der Assoziation in vier Subass.: typicum, quercetosum sessiliflorae, quercetosum pubescentis und carpinetosum betuli angegeben.

Späterhin wird die passende Namensform „Seslerio-Ostryetum Horvat et Horvatić apud Horvat 1950“ angenwandt mit den Subassoziationen: quercetosum pubescentis, quercetosum petaeae, sorbetosum ariae und carpinetosum betuli (Horvat 1962).

Erst in 1959 erscheint eine aus 6 Aufnahmen bestehende synthetische Tabelle von Seslerio-Ostryetum quercetosum pubescentis (Horvat 1959), die dann von Horvat et al. (1974) weiter benutzt wird.

Das gültige Veröffentlichungsjahr des Namens „Ostryo-Quercetum pubescentis“ sollte 1977 sein, weil erst in diesem Jahr Trinajstić eine synthetische Tabelle der neubenannten Assoziation publiziert. Eine andere synthetische Tabelle erscheint im 1987. Das vom Autor selbst angeführte Jahr 1974 kann nicht als valides Veröffentlichungsjahr gelten, weil die dieses Datum tragende Arbeit weder Aufnahmen noch synthetische Tabelle der Assoziation enthält.

Trinajstić selber, bewußt, dass der Name „OstryoQuercetum pubescentis" nicht anwendbar ist, weil es ein späteres Synonym von Querco pubescentis-Ostryetum Horvat 1938, dem wärmeliebenden Hopfenbuchen-Flaumeichenwald auf Dolomit im kontinentalen Kroatien ist (Art. 32d), stellt den neuen Namen Seslerio autumnalis-Quercetum pubescentis Trinajstić 2008 auf, der aber schon von Zupančič (1999) gebraucht wurde für einen auf Flysch-Unterlage wachsenden Wald im submediterranen Bereich Sloweniens.

Aufgrund dieser Erwägungen ergibt sich die Notwendigkeit einen neuen Namen und einen Neotypus aufzustellen:

Aristolochio luteae-Quercetum pubescentis (Horvat 1959) Poldini, nom. nov. hoc loco

Neotypus: rel. 4, tab. 1 ex Trinajstić 1982, Art. 21.

Basionym: Seslerio-Ostryetum quercetosum pubescentis Horvat 1959, Art. 7

\footnotetext{
* Department of Life Sciences, University of Trieste, Via L. Giorgieri 10, I-34127 Trieste, Italy, poldini@units.it
} 
Syn.: Ostryo-Quercetum pubescentis (Horvat 1959) Trinajstić 1974, Art 32d; Seslerio autumnalis-Quercetum pubescentis Trinajstić 2008 non Zupančič 1999, Art. 31.

In der Tabelle von Trinajstić, aus der der Neotypus entnommen wird, erscheint noch Aristolochia pallida unter den Kennarten, aber in einer weiteren Arbeit des Autors über A. pallida agg. in Kroatien (Trinajstić 1990) stellt es sich heraus, dass in den Lokalitäten der Aufnahmen (Hvar, Korčula, Vis, Mljet, Brač) nur A. lutea vorkommt.

\section{DANKSAGUNG}

Für nomenklatorische Beratung und Literaturbeschaffung bin ich Prof. J.-P. Theurillat (Champex) und Prof. T. Wraber (Ljubljana) verbindlichst dankbar.

\section{LITERATURVERZEICHNIS}

Horvat, I. 1959: Sistematski odnosi termofilnih hrastovih i borovih šuma Jugoistočne Evrope. Biološki glasnik 12: 1-40.

Horvat, I. 1962: Vegetacija planina Zapadne Hrvatske. Prirodoslovna istraživanja 30 , Acta Biologica 2: 116-117.

Horvat, I., Glavač, V., Ellenberg, H. 1974: Vegetation Südosteuropas. Vegetation of Southeast-Europe. Gustav Fischer Verlag, Stuttgart, 768 pp.
Trinajstić, I. 1974: Novi pogledi na fitogeografsko rasčlanjavanje vegetacije Jadranskog Primorja Jugoslavije (Some new views of the phytogeographical division of the Adriatic littoral vegetation in Yugoslavia). IV. Kongres biologa Jugoslavije, Sarajevo 25.-28. juna '74, Rezimei referata: 46-47.

Trinajstić, I. 1977: O vegetacijskoj granici mediteranske regije na primorskoj padini Dinarida. (Über die Vegetationsgrenze der mediterranen Region auf dem Küstenabhang der Dinariden). Poljoprivreda i šumarstvo 23(1): 1-11.

Trinajstić, I. 1982: Die Bedeutung der Hopfenbuche Ostrya carpinifolia Scop. für die pflanzengeographische Begrenzung der mediterran-montanen Vegetationsstufe auf den adriatischen Inseln. Studia Geobotanica 2: 7-14.

Trinajstić, I. 1987: Sulla sintassonomia delle vegetazioni termofile caducifoglie dell'ordine Quercetalia pubescentis $\mathrm{Br}$.-Bl. del litorale adriatico jugoslavo. Notiziario della Società Italiana di Fitosociologia 23: 21-28.

Trinajstić, I. 1990: Aristolochia pallida Willd. (Aristolochiaceae) in the Flora of Croatia. Acta Botanica Croatica 49: 143-146.

Trinajstić, I. 2008: Biljne zajednice Republike Hrvatske. (Plant Communities of Croatia). Akademija šumarskih znanosti, Zagreb, 179 pp.

Zupančič, M. 1999: Novosti o gozdno-grmiščni vegetaciji slovenskega submediterana. (Some news about forest-shrub vegetation of Slovenia's Submediterranean). Razprave IV. razreda SAZU 40(8): 196-313. 